ESAIM: PROCEEDINGS AND SURVEYS, September 2014, Vol. 45, p. 429-436

J.-S. Dhersin, Editor

\title{
ON THE EQUIVALENCE OF FINANCIAL STRUCTURES WITH SHORT-TERM ASSETS
}

\author{
Achis Chery ${ }^{1}$ and Jean-Marc Bonnisseau ${ }^{2}$
}

\begin{abstract}
Two financial structures are equivalent if, for each given state price, the images of their full payoff matrices of these financial structures are equal. The main consequence of this definition is that, regardless of the standard exchange economy $\Sigma$, the existence of a financial equilibrium in an exchange economy $\Sigma$ associated with a financial structure $\mathcal{F}$ is equivalent to the existence of an equilibrium in $\Sigma$ associated with any other financial structure $\mathcal{F}^{\prime}$ belonging to the equivalence class of $\mathcal{F}$. The main contribution of the paper is to provide a necessary and sufficient condition of equivalence in a multi-period economy if all assets are short-term.

Résumé. Deux structures financières sont équivalentes si, pour chaque prix d'état donné, les images de leurs matrices de paiement sont égales. La principale conséquence de cette définition est que, quel que soit l'économie d'échange standard $\Sigma$, l'existence d'un équilibre financier dans cette économie d'échange $\Sigma$ associée à une structure financière $\mathcal{F}$ est équivalent à l'existence d'un équilibre dans $\Sigma$ associée avec n'importe quelle autre structure financière $\mathcal{F}^{\prime}$ appartenant à la classe d'équivalence de $\mathcal{F}$. La principale contribution de ce papier est de produire une condition nécessaire et suffisante d'équivalence dans une économie à plusieurs périodes si tous les actifs sont de court terme.
\end{abstract}

\section{INTRODUCTION}

We consider a stochastic financial exchange economy with a finite date-event tree representing time and uncertainty and a financial structure with a finite number of assets.

We say that two financial structures are equivalent if, for each given state price, the images of their full payoff matrices are equal. The intuition behind this definition is that the financial structures allow agents to transfer wealth across nodes of the date-event tree. Thereby two equivalent financial structures offer the same opportunities to the agents. The main consequence of this definition is that, regardless of the standard exchange economy $\Sigma$, the existence of equilibrium in an exchange economy $\Sigma$ associated with a financial structure $\mathcal{F}$ is equivalent to the existence of equilibrium in $\Sigma$ associated with any other financial structure $\mathcal{F}^{\prime}$ belonging to the equivalence class of $\mathcal{F}$. In this case, the equilibrium consumption and the equilibrium spot price are the same.

The equivalence relation has been studied, among others, by Aouani-Cornet and Cornet-Ranjan $[2,4]$ in the two-period case. The purpose of this paper is to extend their result to a multi-period financial structure with short-term assets, which deliver non-zero payoffs only at the successors of their issuance node. Precisely, we

\footnotetext{
1 Paris School of Economics, Université Paris 1 Panthéon Sorbonne, 106-112 Boulevard de l'Hôpital, 75647 Paris Cedex 13, France et Centre de Recherche en Gestion et Economie du Développement (CREGED), Université Quisqueya, 218 Haut Turgeau, 6113 Port-au-prince, Haïti, Achis.Chery@malix.univ-paris1.fr

2 Paris School of Economics, Université Paris 1 Panthéon Sorbonne, 106-112 Boulevard de l'Hôpital, 75647 Paris Cedex 13, France, Jean-marc.Bonnisseau@univ-paris1.fr
}

(C) EDP Sciences, SMAI 2014 
prove that two financial structures with short term assets are equivalent if and only if the images of the payoff matrices are equal.

Note that the result is no more true with long-term assets where the equality of the images of payoff matrices is neither a sufficient condition nor a sufficient one. This is illustrated in examples in [3] (see Remarks 4.1 and $4.2)$.

\section{Financial EXChange ECONOMY AND EQUILIBRIUM}

In this section, we present the model and the notations, which are borrowed from Angeloni-Cornet [1] and are essentially the same as those of Magill-Quinzii [5].

\subsection{Time and uncertainty}

$\mathrm{We}^{1}$ consider a multi-period exchange economy with $(T+1)$ dates, $t \in \mathcal{T}:=\{0, \ldots, T\}$, and a finite set of agents $\mathcal{I}$. The uncertainty is described by a date-event tree $\mathbb{D}$ of length $T+1$. The set $\mathbb{D}_{t}$ is the set of nodes (also called date-events) that could occur at date $t$ and the family $\left(\mathbb{D}_{t}\right)_{t \in \mathcal{T}}$ defines a partition of the set $\mathbb{D}$; for each $\xi \in \mathbb{D}$, we denote by $t(\xi)$ the unique date $t \in \mathcal{T}$ such that $\xi \in \mathbb{D}_{t}$. At date $t=0$, there is a unique node $\xi_{0}$, that is $\mathbb{D}_{0}=\left\{\xi_{0}\right\}$. As $\mathbb{D}$ is a tree, each node $\xi$ in $\mathbb{D} \backslash\left\{\xi_{0}\right\}$ has a unique immediate predecessor denoted $\operatorname{pr}(\xi)$ or $\xi^{-}$. The mapping $p r$ maps $\mathbb{D}_{t}$ to $\mathbb{D}_{t-1}$. Each node $\xi \in \mathbb{D} \backslash \mathbb{D}_{T}$ has a set of immediate successors defined by $\xi^{+}=\left\{\bar{\xi} \in \mathbb{D}: \xi=\bar{\xi}^{-}\right\}$. For $\tau \in \mathcal{T} \backslash\{0\}$ and $\xi \in \mathbb{D} \backslash \cup_{t=0}^{\tau-1} \mathbb{D}_{t}$, we define $\operatorname{pr}^{\tau}(\xi)$ by the recursive formula: $p r^{\tau}(\xi)=p r\left(p r^{\tau-1}(\xi)\right)$. We then define the set of successors and the set of predecessors of $\xi$ as follows:

$$
\begin{aligned}
& \mathbb{D}^{+}(\xi)=\left\{\xi^{\prime} \in \mathbb{D}: \exists \tau \in \mathcal{T} \backslash\{0\} \mid \xi=p r^{\tau}\left(\xi^{\prime}\right)\right\} \\
& \mathbb{D}^{-}(\xi)=\left\{\xi^{\prime} \in \mathbb{D}: \exists \tau \in \mathcal{T} \backslash\{0\} \mid \xi^{\prime}=p r^{\tau}(\xi)\right\}
\end{aligned}
$$

For each $\xi \in \mathbb{D}$, we note by $\mathbb{D}(\xi)$ the union of $\xi$ with $\mathbb{D}^{+}(\xi)$ i.e., $\mathbb{D}(\xi):=\mathbb{D}^{+}(\xi) \cup\{\xi\}$. If $\xi^{\prime} \in \mathbb{D}^{+}(\xi)$ [resp. $\left.\xi^{\prime} \in \mathbb{D}^{+}(\xi) \cup\{\xi\}\right]$, we shall use the notation $\xi^{\prime}>\xi\left[\right.$ resp. $\left.\xi^{\prime} \geq \xi\right]$. Note that $\xi^{\prime} \in \mathbb{D}^{+}(\xi)$ if and only if $\xi \in \mathbb{D}^{-}\left(\xi^{\prime}\right)$ and similarly $\xi^{\prime} \in \xi^{+}$if and only if $\xi=\left(\xi^{\prime}\right)^{-}$.

\subsection{The financial structure}

The financial structure is constituted by a finite set of assets denoted $\mathcal{J}=\{1, \ldots, J\}$. An asset $j \in \mathcal{J}$ is a contract issued at a given and unique node in $\mathbb{D}$ denoted $\xi(j)$, called issuance node of $j$. Each asset is bought or sold only at its issuance node $\xi(j)$ and yields payoffs only at the successor nodes $\xi^{\prime}$ of $\mathbb{D}^{+}(\xi(j))$. To simplify the notation, we consider the payoff of asset $j$ at every node $\xi \in \mathbb{D}$ and we assume that it is zero if $\xi$ is not a successor of the issuance node $\xi(j)$. The payoff may depend upon the spot price vector ${ }^{2} p \in \mathbb{R}^{\mathbb{L}}$ and is denoted by $V_{\xi}^{j}(p)$. Formally, we assume that $V_{\xi}^{j}(p)=0$ if $\xi \notin \mathbb{D}^{+}(\xi(j))$. An asset is a short term asset if it has a non-zero payoff only at the immediate successors of the issuance node, that is, $V_{\xi^{\prime}}^{j}(p)=0$ if $\xi^{\prime} \notin \xi^{+}$. In the following, we consider only non trivial assets, that is assets having a non zero return in at least one node.

A portfolio $z=\left(z^{j}\right)_{j \in \mathcal{J}}$ is an element of $\mathbb{R}^{\mathcal{J}}$. If $z^{j}>0$ [resp. $\left.z^{j}<0\right]$, then $\left|z^{j}\right|$ is the quantity of asset $j$ bought [resp. sold] at the issuance node $\xi(j)$.

To summarize a financial structure $\mathcal{F}=\left(\mathcal{J},(\xi(j))_{j \in \mathcal{J}}, V\right)$ consists of:

(1) a set of non trivial assets $\mathcal{J}$,

\footnotetext{
${ }^{1}$ We use the following notations. A $(\mathbb{D} \times \mathcal{J})$-matrix $A$ is an element of $\mathbb{R}^{\mathbb{D} \times \mathcal{J}}$, with entries $\left(a_{\xi}^{j}\right)_{(\xi \in \mathbb{D}, j \in \mathcal{J})}$; we denote by $A_{\xi} \in \mathbb{R}^{\mathcal{J}}$ the $\xi$-th row of $A$ and by $A^{j} \in \mathbb{R}^{\mathbb{D}}$ the $j$-th column of $A$. We recall that the transpose of $A$ is the unique $(\mathcal{J} \times \mathbb{D})$-matrix ${ }^{t} \mathrm{~A}$ satisfying $(A x) \bullet \mathbb{D} y=x \bullet \mathcal{J}\left({ }^{t}\right.$ Ay) for every $x \in \mathbb{R}^{\mathcal{J}}, y \in \mathbb{R}^{\mathbb{D}}$, where $\bullet \mathbb{D}$ [resp. $\bullet \mathcal{J}$ ] denotes the usual inner product in $\mathbb{R}^{\mathbb{D}}\left[\right.$ resp. $\left.\mathbb{R}^{\mathcal{J}}\right]$. We denote by $\operatorname{rank} A$ the $\operatorname{rank}$ of the matrix $A$ and by $\operatorname{im}(A)$ the range of the matrix $A$, that is the linear sub-space spanned by the column vectors of $A$. For every subset $\tilde{\mathbb{D}} \subset \mathbb{D}$ and $\tilde{\mathcal{J}} \subset \mathcal{J}$, the matrix $A^{\tilde{\mathcal{J}}_{\tilde{\mathbb{D}}}}$ is the $(\tilde{\mathbb{D}} \times \tilde{\mathcal{J}})$-sub-matrix of $A$ with entries $a_{\xi}^{j}$ for every $(\xi, j) \in(\tilde{\mathbb{D}} \times \tilde{\mathcal{J}})$. Let $x, y$ be in $\mathbb{R}^{n} ; x \geq y$ (resp. $\left.x \gg y\right)$ means $x_{h} \geq y_{h}$ (resp. $x_{h}>y_{h}$ ) for every $h=1, \ldots, n$ and we let $\mathbb{R}_{+}^{n}=\left\{x \in \mathbb{R}^{n}: x \geq 0\right\}, \mathbb{R}_{++}^{n}=\left\{x \in \mathbb{R}^{n}: x \gg 0\right\}$. We also use the notation $x>y$ if $x \geq y$ and $x \neq y$.

${ }^{2} \mathbb{L}=\mathbb{H} \times \mathbb{D}$ where $\mathbb{H}$ is a finite set of divisible and physical goods exchanged at each node $\xi \in \mathbb{D}$.
} 
(2) a payoff mapping $V: \mathbb{R}^{\mathbb{L}} \rightarrow \mathbb{R}^{\mathbb{D} \times \mathcal{J}}$ which associates to every spot price $p \in \mathbb{R}^{\mathbb{L}}$ the $(\mathbb{D} \times \mathcal{J})$-payoff matrix $V(p)=\left(V_{\xi}^{j}(p)\right)_{\xi \in \mathbb{D}, j \in \mathcal{J}}$ and satisfies the condition $V_{\xi}^{j}(p)=0$ if $\xi \notin \mathbb{D}^{+}(\xi(j))$.

The price of asset $j$ is denoted by $q_{j}$; it is paid at its issuance node $\xi(j)$. We let $q=\left(q_{j}\right)_{j \in \mathcal{J}} \in \mathbb{R}^{\mathcal{J}}$ be the asset price vector.

The full payoff matrix $W(p, q)$ is the $(\mathbb{D} \times \mathcal{J})$-matrix with the following entries:

$$
W_{\xi}^{j}(p, q):=V_{\xi}^{j}(p)-\delta_{\xi, \xi(j)} q_{j},
$$

where $\delta_{\xi, \xi^{\prime}}=1$ if $\xi=\xi^{\prime}$ and $\delta_{\xi, \xi^{\prime}}=0$ otherwise.

So, given the prices $(p, q)$, the full flow of returns for a given portfolio $z \in \mathbb{R}^{\mathcal{J}}$ is $W(p, q) z$ and the full return at node $\xi$ is

$$
\begin{gathered}
{[W(p, q) z](\xi):=W_{\xi}(p, q) \bullet \mathcal{J} z=\sum_{j \in \mathcal{J}} V_{\xi}^{j}(p) z_{j}-\sum_{j \in \mathcal{J}} \delta_{\xi, \xi(j)} q_{j} z_{j}} \\
=\sum_{\{j \in \mathcal{J} \mid \xi(j)<\xi\}} V_{\xi}^{j}(p) z_{j}-\sum_{\{j \in \mathcal{J} \mid \xi(j)=\xi\}} q_{j} z_{j},
\end{gathered}
$$

We now recall that for a given spot price $p$, the asset price $q$ is an arbitrage free price if it does not exist a portfolio $z \in \mathbb{R}^{\mathcal{J}}$ such that $W(p, q) z>0 . q$ is an arbitrage free price if and only if it exists a so-called state price vector $\lambda \in \mathbb{R}_{++}^{\mathbb{D}}$ such that ${ }^{t} W(p, q) \lambda=0$ (see, e.g. Magill-Quinzii [5]). Taken into account the particular structure of the matrix $W(p, q)$, this is equivalent to

$$
\forall j \in \mathcal{J}, \lambda_{\xi(j)} q_{j}=\sum_{\xi \in \mathbb{D}^{+}(\xi(j))} \lambda_{\xi} V_{\xi}^{j}(p) .
$$

\section{Some additional notations}

For all $\xi \in \mathbb{D} \backslash \mathbb{D}_{T}, \mathcal{J}(\xi)$ is the set of assets issued at the node $\xi$, that is $\mathcal{J}(\xi)=\{j \in \mathcal{J} \mid \xi(j)=\xi\}$, $\mathbb{D}^{e}$ is the set of nodes at which there is the issuance of at least one asset.

If $\xi \notin \mathbb{D}^{e}, \mathcal{J}(\xi)=\emptyset$ and, by convention, we let $\sum_{j \in \mathcal{J}(\xi)} V^{j}(p)=0$ and for all arbitrage free price $q$ we have $\sum_{j \in \mathcal{J}(\xi)} W^{j}(p, q)=0$.

\subsection{The stochastic exchange economy}

At each node $\xi \in \mathbb{D}$, there is a spot market on which a finite set $\mathbb{H}=\{1, \ldots, H\}$ of divisible and physical goods are exchanged. We assume that each good is perishable, that is, its life does not have more than one date. In this model, a commodity is a pair $(h, \xi)$ of a physical good $h \in \mathbb{H}$ and the node $\xi \in \mathbb{D}$ at which the good is available. Then the commodity space is $\mathbb{R}^{\mathbb{L}}$, where $\mathbb{L}=\mathbb{H} \times \mathbb{D}$. An element $x \in \mathbb{R}^{\mathbb{L}}$ is called a consumption, that is to say $x=(x(\xi))_{\xi \in \mathbb{D}} \in \mathbb{R}^{\mathbb{L}}$, where $x(\xi)=(x(h, \xi))_{h \in \mathbb{H}} \in \mathbb{R}^{\mathbb{H}}$ for each $\xi \in \mathbb{D}$.

We denote by $p=(p(\xi))_{\xi \in \mathbb{D}} \in \mathbb{R}^{\mathbb{L}}$ the vector of spot prices and $p(\xi)=(p(h, \xi))_{h \in \mathbb{H}} \in \mathbb{R}^{\mathbb{H}}$ is called the spot price at node $\xi$. The spot price $p(h, \xi)$ is the price at the node $\xi$ for immediate delivery of one unit of the physical good $h$. Thus the value of a consumption $x(\xi)$ at node $\xi \in \mathbb{D}$ (measured in unit account of the node $\xi$ ) is

$$
p(\xi) \bullet \mathbb{H} x(\xi)=\sum_{h \in \mathbb{H}} p(h, \xi) x(h, \xi) .
$$

We consider a finite set of consumers $\mathcal{I}=\{1, \ldots, I\}$. Each agent $i \in \mathcal{I}$ has a consumption set $X_{i} \subset \mathbb{R}^{\mathbb{L}}$, which consists of all possible consumptions. An allocation is an element $x \in \prod_{i \in \mathcal{I}} X_{i}$ and we denote by $x_{i}$ the consumption of agent $i$, which is the projection of $x$ on $X_{i}$.

The tastes of each consumer $i \in \mathcal{I}$ are represented by a strict preference correspondence $P_{i}: \prod_{j \in \mathcal{I}} X_{j} \longrightarrow X_{i}$, where $P_{i}(x)$ defines the set of consumptions that are strictly preferred to $x_{i}$ for agent $i$, given the consumption 
$x_{j}$ for the other consumers $j \neq i . P_{i}$ represents the consumer tastes, but also his behavior with respect to time and uncertainty, especially his impatience and attitude toward risk. If consumer preferences are represented by utility functions $u_{i}: X_{i} \longrightarrow \mathbb{R}$ for each $i \in \mathcal{I}$, the strict preference correspondence is defined by $P_{i}(x)=$ $\left\{\bar{x}_{i} \in X_{i} \mid u_{i}\left(\bar{x}_{i}\right)>u_{i}\left(x_{i}\right)\right\}$.

Finally, for each node $\xi \in \mathbb{D}$, every consumer $i \in \mathcal{I}$ has a node endowment $e_{i}(\xi) \in \mathbb{R}^{\mathbb{H}}$ (contingent on the fact that $\xi$ prevails) and we denote by $e_{i}=\left(e_{i}(\xi)\right)_{\xi \in \mathbb{D}} \in \mathbb{R}^{\mathbb{L}}$ the endowments for the whole set of nodes. The exchange economy $\Sigma$ can be summarized by

$$
\Sigma=\left[\mathbb{D}, \mathbb{H}, \mathcal{I},\left(X_{i}, P_{i}, e_{i}\right)_{i \in \mathcal{I}}\right] .
$$

\subsection{Financial equilibrium}

We now consider a financial exchange economy, which is defined as the couple of an exchange economy $\Sigma$ and a financial structure $\mathcal{F}$. It can thus be summarized by

$$
(\Sigma, \mathcal{F}):=\left[\mathbb{D}, \mathbb{H}, \mathcal{I},\left(X_{i}, P_{i}, e_{i}\right)_{i \in \mathcal{I}}, \mathcal{J},(\xi(j))_{j \in \mathcal{J}}, V\right] .
$$

Given the price $(p, q) \in \mathbb{R}^{\mathbb{L}} \times \mathbb{R}^{\mathcal{J}}$, the budget set of consumer $i \in \mathcal{I}$ is $B_{\mathcal{F}}^{i}(p, q)$ defined by ${ }^{3}$ :

$$
\left\{\left(x_{i}, z_{i}\right) \in X_{i} \times \mathbb{R}^{\mathcal{J}}: \forall \xi \in \mathbb{D}, p(\xi) \bullet \mathbb{H}\left[x_{i}(\xi)-e_{i}(\xi)\right] \leq\left[W(p, q) z_{i}\right](\xi)\right\}
$$

or

$$
\left\{\left(x_{i}, z_{i}\right) \in X_{i} \times \mathbb{R}^{\mathcal{J}}: p \square\left(x_{i}-e_{i}\right) \leq W(p, q) z_{i}\right\} .
$$

We now introduce the equilibrium notion:

Definition 1.1. An equilibrium of the financial exchange economy $(\Sigma, \mathcal{F})$ is a list of strategies and prices $(\bar{x}, \bar{z}, \bar{p}, \bar{q}) \in\left(\mathbb{R}^{\mathbb{L}}\right)^{\mathcal{I}} \times\left(\mathbb{R}^{\mathcal{J}}\right)^{\mathcal{I}} \times \mathbb{R}^{\mathbb{L}} \backslash\{0\} \times \mathbb{R}^{\mathcal{J}}$ such that

(a): for every $i \in \mathcal{I},\left(\bar{x}_{i}, \bar{z}_{i}\right)$ maximizes the preferences $P_{i}$ in the budget set $B_{\mathcal{F}}^{i}(\bar{p}, \bar{q})$, in the sense that

$$
\left(\bar{x}_{i}, \bar{z}_{i}\right) \in B_{\mathcal{F}}^{i}(\bar{p}, \bar{q}) \text { and }\left[P_{i}(\bar{x}) \times \mathbb{R}^{\mathcal{J}}\right] \bigcap B_{\mathcal{F}}^{i}(\bar{p}, \bar{q})=\emptyset
$$

(b): $\sum_{i \in \mathcal{I}} \bar{x}_{i}=\sum_{i \in \mathcal{I}} e_{i}$ and $\sum_{i \in \mathcal{I}} \bar{z}_{i}=0$.

We recall that the equilibrium portfolios are arbitrage free under the following Non-Satiation Assumption:

Assumption NS (i) For every $\bar{x} \in \prod_{i \in \mathcal{I}} X_{i}$ such that $\sum_{i \in \mathcal{I}} \bar{x}_{i}=\sum_{i \in \mathcal{I}} e_{i}$, (Non-Saturation at Every Node) for every $i \in \mathcal{I}$, for every $\xi \in \mathbb{D}$, there exists $x_{i} \in X_{i}$ such that, for each $\xi^{\prime} \neq \xi, x_{i}\left(\xi^{\prime}\right)=\bar{x}_{i}\left(\xi^{\prime}\right)$ and $x_{i} \in P_{i}(\bar{x})$. (ii) if $x_{i} \in P_{i}(\bar{x})$, then $\left[x_{i}, \bar{x}_{i}\left[\in P_{i}(\bar{x})\right.\right.$.

Proposition 1.2. (Angeloni-Cornet [1]) Under $(\boldsymbol{N S})$, if $(\bar{x}, \bar{z}, \bar{p}, \bar{q})$ is an equilibrium of the economy $(\Sigma, \mathcal{F})$, then the financial structure $\mathcal{F}$ is arbitrage free at $(\bar{p}, \bar{q})$ i.e., there exists a state price $\lambda \in \mathbb{R}_{++}^{\mathbb{D}}$ such that ${ }^{t} W_{\mathcal{F}}(\bar{p}, \bar{q}) \lambda=0$.

\section{Equivalent financial Structures}

In this section we will define an equivalence relation on financial structures. We will show that the existence of an equilibrium in an exchange economy associated with a given financial structure is equivalent to the existence of equilibrium in exchange economy associated with any other financial structure equivalent to the first one. So equivalence allows to extend the existence results for financial equilibrium to a whole class of financial structures. Hence the importance of studying the notion of equivalence between the financial structures.

\footnotetext{
${ }^{3}$ For $x=(x(\xi))_{\xi \in \mathbb{D}}, p=(p(\xi))_{\xi \in \mathbb{D}}$ in $\mathbb{R}^{\mathbb{L}}=\mathbb{R}^{\mathbb{H} \times \mathbb{D}}\left(\right.$ with $x(\xi), p(\xi)$ in $\left.\mathbb{R}^{\mathbb{H}}\right)$ we let $p \square x=(p(\xi) \bullet \mathbb{H} x(\xi))_{\xi \in \mathbb{D}} \in \mathbb{R}^{\mathbb{D}}$.
} 
Definition 2.1. Given a spot price vector $p \in \mathbb{R}^{\mathbb{L}}$ let $\mathcal{F}_{1}=\left(\mathcal{J}_{1},(\xi(j))_{j \in \mathcal{J}_{1}}, V^{1}\right)$ and $\mathcal{F}_{2}=\left(\mathcal{J}_{2},(\xi(j))_{j \in \mathcal{J}_{2}}, V^{2}\right)$ be two financial structures. We say that $\mathcal{F}_{1}$ is equivalent to $\mathcal{F}_{2}$ with respect to $p$ ( we denote by $\mathcal{F}_{1} \simeq p \mathcal{F}_{2}$ ) if for all state price $\lambda=\left(\lambda_{\xi}\right)_{\xi \in \mathbb{D}} \in \mathbb{R}_{++}^{\mathbb{D}}$, im $W^{1}\left(p, q^{1}\right)=\operatorname{im} W^{2}\left(p, q^{2}\right)$ where $q^{1} \in \mathbb{R}^{\mathcal{J}_{1}}\left[\operatorname{resp}\right.$. $\left.q^{2} \in \mathbb{R}^{\mathcal{J}_{2}}\right]$ is the arbitrage free price of the financial structure $\mathcal{F}_{1}$ [resp. $\left.\mathcal{F}_{2}\right]$ associated with $\lambda$ i.e., ${ }^{t} W^{1}\left(p, q^{1}\right) \lambda=0[$ resp. $\left.{ }^{t} W^{2}\left(p, q^{2}\right) \lambda=0\right]$.

We say that $\mathcal{F}_{1}$ is equivalent to $\mathcal{F}_{2}$ if for all spot price vector $p \in \mathbb{R}^{\mathbb{L}}$, we have $\mathcal{F}_{1} \simeq_{p} \mathcal{F}_{2}$.

The intuition behind this definition is that the financial structures allow agents to transfer wealth across nodes of the date-event tree. Thereby given a spot price $p$, their budget set is determined by the image of the full payoff matrix. The main consequence of this definition is given below and states that, regardless of the standard exchange economy $\Sigma$, consumption equilibria are the same when agents carry out their financial activities through two different equivalence structures $\mathcal{F}_{1}$ and $\mathcal{F}_{2}$ with no portfolio constraints.

Proposition 2.2. Let $\Sigma$ be an exchange economy satisfying Assumption $\mathbf{N S}$.

Let $\mathcal{F}_{1}=\left(\mathcal{J}_{1},(\xi(j))_{j \in \mathcal{J}_{1}}, V^{1}\right)$ and $\mathcal{F}_{2}=\left(\mathcal{J}_{2},(\xi(j))_{j \in \mathcal{J}_{2}}, V^{2}\right)$ be two equivalent financial structures with respect to the spot price $\bar{p} \in \mathbb{R}^{\mathbb{L}}$.

Let $\left(\bar{x}, \bar{z}, \bar{p}, \bar{q}^{1}\right)$ be an equilibrium of $\left(\Sigma, \mathcal{F}_{1}\right)$. Then there exists $\hat{z}$ and $\bar{q}^{2}$ such that $\left(\bar{x}, \hat{z}, \bar{p}, \bar{q}^{2}\right)$ is an equilibrium of $\left(\Sigma, \mathcal{F}_{2}\right)$.

Proof. Let $\left(\bar{x}, \bar{z}, \bar{p}, \bar{q}^{1}\right)$ be an equilibrium of $\left(\Sigma, \mathcal{F}_{1}\right)$. Since $\left(\bar{x}, \bar{z}, \bar{p}, \bar{q}^{1}\right)$ is an equilibrium and Assumption NS is satisfied, $\bar{q}^{1}$ is an arbitrage free price i.e., there exists a state price $\lambda=\left(\lambda_{\xi}\right)_{\xi \in \mathbb{D}} \in \mathbb{R}_{++}^{\mathbb{D}}$ which satisfies ${ }^{t} W^{1}\left(\bar{p}, \bar{q}^{1}\right) \lambda=0$. Let $\bar{q}^{2}$ be the arbitrage free price for the financial structure $\mathcal{F}_{2}$ associed with $\lambda$ i.e., ${ }^{t} W^{2}\left(\bar{p}, \bar{q}^{2}\right) \lambda=0$. Since $\mathcal{F}_{1} \simeq{ }_{p} \mathcal{F}_{2}$, we have $\operatorname{im} W^{1}\left(p, q^{1}\right)=\operatorname{im} W^{2}\left(p, q^{2}\right)$ (See Definition 2.1).

Let $\hat{z} \in \mathbb{R}^{\mathcal{J}_{2} \mathcal{I}}$ be such that $W^{1}\left(\bar{p}, \bar{q}^{1}\right) \bar{z}_{i}=W^{2}\left(\bar{p}, \bar{q}^{2}\right) \hat{z}_{i}$ for all $i \neq 1$. Such $\hat{z}$ exists because im $W^{1}\left(p, q^{1}\right)=$ $\operatorname{im} W^{2}\left(p, q^{2}\right)$. Let $\hat{z}_{1}=-\sum_{i \in \mathcal{I} ; i \neq 1} \hat{z}_{i}$. We now show that $\left(\bar{x}, \hat{z}, \bar{p}, \bar{q}^{2}\right)$ is an equilibrium of $\left(\Sigma, \mathcal{F}_{2}\right)$. Indeed, let $i \in \mathcal{I}$, we have $\left(\bar{x}_{i}, \hat{z}_{i}\right) \in B_{\mathcal{F}_{2}}^{i}\left(\bar{p}, \bar{q}^{2}\right)$ because, by definition of $\hat{z}_{i}$, if $i \neq 1$

$$
W^{2}\left(\bar{p}, \bar{q}^{2}\right) \hat{z}_{i}=W^{1}\left(\bar{p}, \bar{q}^{1}\right) \bar{z}_{i}
$$

and if $i=1$, as $\sum_{i \in \mathcal{I}} \bar{z}_{i}=0$,

$$
\begin{gathered}
W^{2}\left(\bar{p}, \bar{q}^{2}\right) \hat{z}_{1}=W^{2}\left(\bar{p}, \bar{q}^{2}\right)\left(-\sum_{i \in \mathcal{I} ; i \neq 1} \hat{z}_{i}\right)=-\sum_{i \in \mathcal{I} ; i \neq 1}\left[W^{2}\left(\bar{p}, \bar{q}^{2}\right) \hat{z}_{i}\right]= \\
-\sum_{i \in \mathcal{I} ; i \neq 1}\left[W^{1}\left(\bar{p}, \bar{q}^{1}\right) \bar{z}_{i}\right]=W^{1}\left(\bar{p}, \bar{q}^{1}\right)\left(-\sum_{i \in \mathcal{I} ; i \neq 1} \bar{z}_{i}\right)=W^{1}\left(\bar{p}, \bar{q}^{1}\right) \bar{z}_{1}
\end{gathered}
$$

thus for all $i \in \mathcal{I}$ and $\xi \in \mathbb{D}$,

$$
\bar{p}(\xi) \bullet \mathbb{H}\left[\bar{x}_{i}(\xi)-e_{i}(\xi)\right] \leq\left[W^{1}\left(\bar{p}, \bar{q}^{1}\right) \bar{z}_{i}\right](\xi)=\left[W^{2}\left(\bar{p}, \bar{q}^{2}\right) \hat{z}_{i}\right](\xi)
$$

For all $i \in \mathcal{I},\left[P_{i}(\bar{x}) \times \mathbb{R}^{\mathcal{J}_{2}}\right] \cap B_{\mathcal{F}_{2}}^{i}\left(\bar{p}, \bar{q}^{2}\right)=\emptyset$. Let us argue by contradiction. Suppose that there exists $i \in \mathcal{I}$ and $\left(x_{i}, z_{i}\right)$ such that

$$
\left(x_{i}, z_{i}\right) \in\left[P_{i}(\bar{x}) \times \mathbb{R}^{\mathcal{J}_{2}}\right] \bigcap B_{\mathcal{F}_{2}}^{i}\left(\bar{p}, \bar{q}^{2}\right)
$$

and $z_{i} \neq \hat{z}_{i}$, this implies that $x_{i} \in P_{i}(\bar{x})$ and for all $\xi \in \mathbb{D}$,

$$
\bar{p}(\xi) \bullet \mathbb{H}\left[x_{i}(\xi)-e_{i}(\xi)\right] \leq\left[W^{2}\left(\bar{p}, \bar{q}^{2}\right) z_{i}\right](\xi) .
$$

But, since im $W^{1}\left(\bar{p}, \bar{q}^{1}\right)=\operatorname{im} W^{2}\left(\bar{p}, \bar{q}^{2}\right)$, there exists $\tilde{z}_{i} \in \mathbb{R}^{\mathcal{J}_{1}}$ such that

$$
W^{1}\left(\bar{p}, \bar{q}^{1}\right) \tilde{z}_{i}=W^{2}\left(\bar{p}, \bar{q}^{2}\right) z_{1}
$$

and this implies that 
$\forall i \in \mathcal{I}, \forall \xi \in \mathbb{D}, \bar{p}(\xi) \bullet \mathbb{H}\left[\bar{x}_{i}(\xi)-e_{i}(\xi)\right] \leq\left[W^{1}\left(\bar{p}, \bar{q}^{1}\right) \tilde{z}_{i}\right](\xi)$

in other words $\left(x_{i}, \tilde{z}_{i}\right) \in B_{\mathcal{F}_{1}}^{i}\left(\bar{p}, \bar{q}^{1}\right)$. As $\left(x_{i}, \tilde{z}_{i}\right) \in\left[P_{i}(\bar{x}) \times \mathbb{R}^{\mathcal{J}_{1}}\right]$, this is in contradiction with the fact that $\left(\bar{x}, \bar{z}, \bar{p}, \bar{q}^{1}\right)$ is an equilibrium financial exchange economy $\left(\Sigma, \mathcal{F}_{1}\right)$.

$\sum_{i \in \mathcal{I}} \bar{x}_{i}=\sum_{i \in \mathcal{I}} e_{i}$, because $\left(\bar{x}, \bar{z}, \bar{p}, \bar{q}^{1}\right)$ is an equilibrium of $\left(\Sigma, \mathcal{F}_{1}\right)$ and $\sum_{i \in \mathcal{I}} \hat{z}_{i}=0$ by definition of $\hat{z}_{i}$. Hence, $\left(\bar{x}, \hat{z}, \bar{p}, \bar{q}^{2}\right)$ is an equilibrium of a financial exchange economy $\left(\Sigma, \mathcal{F}_{2}\right)$.

\subsection{Equivalence of financial structures with short-term assets}

In this section, we state and prove the main result, that is a necessary and sufficient condition for the equivalence of two financial structures consisting of short-term assets. This condition, which is merely the equality of the images of the payoff matrices, extends the well-known result of the two-period case. In [3], we treat the issue of equivalence of financial structures with long-term assets and we also discuss the case of financial structures with re-trading of assets à la Magill-Quinzii. But, contrary to the short-term case, we have only sufficient conditions.

Proposition 2.3 is a generalization of Proposition 6 of Cornet-Ranjan [4].

Proposition 2.3. Given a spot price $p \in \mathbb{R}^{\mathbb{L}}$, let $\mathcal{F}_{1}=\left(\mathcal{J}_{1},(\xi(j))_{j \in \mathcal{J}_{1}}, V^{1}\right)$ and $\mathcal{F}_{2}=\left(\mathcal{J}_{2},(\xi(j))_{j \in \mathcal{J}_{2}}, V^{2}\right)$ be two financial structures consisting only of short-term assets. Then $\mathcal{F}_{1} \simeq_{p} \mathcal{F}_{2} \Leftrightarrow \operatorname{im} V^{1}(p)=\operatorname{im~} V^{2}(p)$.

Proof. Let us assume that im $V^{1}(p)=\operatorname{im} V^{2}(p)$. Let us note by $\mathbb{D}_{1}^{e}\left[\right.$ resp. $\mathbb{D}_{2}^{e}$ ], the set of nodes at which there is the issuance of at least one asset for the financial structure $\mathcal{F}_{1}$ [resp. $\mathcal{F}_{2}$ ].

Step 1. $\mathbb{D}_{1}^{e}=\mathbb{D}_{2}^{e}$.

Indeed let $\xi \in \mathbb{D}_{1}^{e}$ and $j \in \mathcal{J}(\xi)$. Since there are no trivial assets, there exists $V^{1, j}(p) \in \operatorname{im} V^{1}(p), V^{1, j}(p) \neq 0$ and $V_{\eta}^{1, j}(p)=0$ for all $\eta \notin \xi^{+}$. $V^{1, j}(p) \in \operatorname{im} V^{2}(p)$ because im $V^{1}(p)=\operatorname{im} V^{2}(p)$. If $\xi \notin \mathbb{D}_{2}^{e}, V_{\eta}^{2}(p)=0$ for all $\eta \in \xi^{+}$hence $y_{\eta}=0$ for all $y \in \operatorname{im} V^{2}(p)$ and $\eta \in \xi^{+}$. So $V_{\eta}^{1, j}(p)=0$ for all $\eta \in \xi^{+}$. But together with $V_{\eta}^{1, j}(p)=0$ for all $\eta \notin \xi^{+}$, this implies $V^{1, j}(p)=0$, a contradiction. So $\mathbb{D}_{1}^{e} \subset \mathbb{D}_{2}^{e}$. With a similar reasoning we prove that $\mathbb{D}_{2}^{e} \subset \mathbb{D}_{1}^{e}$.

Step 2. $\mathcal{F}_{1} \simeq_{p} \mathcal{F}_{2}$.

Indeed, let $\lambda \in \mathbb{R}_{++}^{\mathbb{D}}$ a given state price and $q^{1}$ the associated arbitrage free asset price satisfying ${ }^{t} W^{1}\left(p, q^{1}\right) \lambda=$ 0 . Let $y \in \operatorname{im} W^{1}\left(p, q^{1}\right)$. There exists $z_{1} \in \mathbb{R}^{\mathcal{J}_{1}}$ such that $y=\sum_{j \in \mathcal{J}_{1}} W^{1, j}\left(p, q^{1}\right) z_{1}^{j}$. Since $\operatorname{im} V^{1}(p)=$ $\operatorname{im} V^{2}(p)$, there exists $z_{2} \in \mathbb{R}^{\mathcal{J}_{2}}$ such that $\sum_{j \in \mathcal{J}_{1}} V^{1, j}(p) z_{1}^{j}=\sum_{j \in \mathcal{J}_{2}} V^{2, j}(p) z_{2}^{j}$. Let us show that $y=$ $\sum_{j \in \mathcal{J}_{1}} W^{1, j}\left(p, q^{1}\right) z_{1}^{j}=\sum_{j \in \mathcal{J}_{2}} W^{2, j}\left(p, q^{2}\right) z_{2}^{j} \in \operatorname{im} W^{2, j}\left(p, q^{2}\right)$ where $q^{2}$ is the arbitrage free asset price associated to $\lambda$ for $\mathcal{F}_{2}$. Let $\xi \in \mathbb{D}$,

If $\xi \notin \mathbb{D}^{e}$ we have $\sum_{j \in \mathcal{J}_{s}} W_{\xi}^{s, j}\left(p, q^{s}\right) z_{s}^{j}=\sum_{j \in \mathcal{J}_{s}} V_{\xi}^{s, j}(p) z_{s}^{j}$ (with $\left.s \in\{1,2\}\right)$ so $y_{\xi}=\sum_{j \in \mathcal{J}_{1}} W_{\xi}^{1, j}\left(p, q^{1}\right) z_{1}^{j}=$ $\sum_{j \in \mathcal{J}_{2}} W_{\xi}^{2, j}\left(p, q^{2}\right) z_{2}^{j}$.

If $\xi \in \mathbb{D}^{e}$ we have $\sum_{j \in \mathcal{J}_{1}} W_{\xi}^{1, j}\left(p, q^{1}\right) z_{1}^{j}=\sum_{j \in \mathcal{J}_{1} \backslash \mathcal{J}_{1}(\xi)} V_{\xi}^{1, j}(p) z_{1}^{j}-\sum_{j \in \mathcal{J}_{1}(\xi)} q_{j}^{1} z_{1}^{j}$ and $\sum_{j \in \mathcal{J}_{2}} W_{\xi}^{2, j}\left(p, q^{2}\right) z_{2}^{j}=$ $\sum_{j \in \mathcal{J}_{2} \backslash \mathcal{J}_{2}(\xi)} V_{\xi}^{2, j}(p) z_{2}^{j}-\sum_{j \in \mathcal{J}_{2}(\xi)} q_{2}^{j} z_{2}^{j}$. Thanks to the structures of the matrices $V^{1}(p)$ and $V^{2}(p)$, we have

$$
\sum_{j \in \mathcal{J}_{s}} V_{\xi}^{s, j}(p) z_{s}^{j}=\sum_{j \in \mathcal{J}_{s} \backslash \mathcal{J}_{s}(\xi)} V_{\xi}^{s, j}(p) z_{s}^{j},(\text { with } s \in\{1,2\}) .
$$

So, to get $\sum_{j \in \mathcal{J}_{1}} W^{1, j}\left(p, q^{1}\right) z_{1}^{j}=\sum_{j \in \mathcal{J}_{2}} W^{2, j}\left(p, q^{2}\right) z_{2}^{j}$, it remains to show that $\sum_{j \in \mathcal{J}_{1}(\xi)} q_{j}^{1} z_{1}^{j}=\sum_{j \in \mathcal{J}_{2}(\xi)} q_{j}^{2} z_{2}^{j}$.

Since the assets are short-term, for every $\xi^{\prime} \in \xi^{+}$, for $s=1,2$,

$$
\sum_{j \in \mathcal{J}_{s}} V_{\xi^{\prime}}^{s, j}(p) z_{s}^{j}=\sum_{j \in \mathcal{J}_{s}(\xi)} V_{\xi^{\prime}}^{s, j}(p) z_{s}^{j} .
$$

So, since $q_{1}$ and $q_{2}$ are arbitrage free prices associated with $\lambda$, 


$$
\begin{aligned}
\sum_{j \in \mathcal{J}_{1}(\xi)} q_{j}^{1} z_{1}^{j} & =\frac{1}{\lambda_{\xi}} \sum_{j \in \mathcal{J}_{1}(\xi)}\left[\left[\sum_{\xi^{\prime} \in \xi^{+}} \lambda_{\xi^{\prime}} V_{\xi}^{1, j}(p)\right] z_{1}^{j}\right] \\
& =\frac{1}{\lambda_{\xi}} \sum_{\xi^{\prime} \in \xi^{+}} \lambda_{\xi^{\prime}}\left[\sum_{j \in \mathcal{J}_{1}(\xi)} V_{\xi}^{1, j}(p) z_{1}^{j}\right] \\
& =\frac{1}{\lambda_{\xi}} \sum_{\xi^{\prime} \in \xi^{+}} \lambda_{\xi^{\prime}}\left[\sum_{j \in \mathcal{J}_{2}(\xi)} V_{\xi}^{2, j}(p) z_{2}^{j}\right] \\
& =\frac{1}{\lambda_{\xi}} \sum_{j \in \mathcal{J}_{2}(\xi)}\left[\left[\sum_{\xi^{\prime} \in \xi^{+}} \lambda_{\xi^{\prime}} V_{\xi}^{2, j}(p)\right] z_{2}^{j}\right] \\
& =\sum_{j \in \mathcal{J}_{2}(\xi)} q_{j}^{2} z_{2}^{j}
\end{aligned}
$$

So, $W_{\xi}^{1}\left(p, q^{1}\right) z^{1}=W_{\xi}^{2}\left(p, q^{2}\right) z^{2}$, which implies $W^{1}\left(p, q^{1}\right) z^{1}=W^{2}\left(p, q^{2}\right) z^{2}$. Hence $y=\sum_{j \in \mathcal{J}_{1}} W^{1, j}\left(p, q^{1}\right) z_{1}^{j}=$ $\sum_{j \in \mathcal{J}_{2}} W^{2, j}\left(p, q^{2}\right) z_{2}^{j} \in W^{2}\left(p, q^{2}\right)$, which proves that $\operatorname{im} W^{1}\left(p, q^{1}\right) \subset \operatorname{im} W^{2}\left(p, q^{2}\right)$. The converse inclusion is obtained by the same argument.

Let us now assume that $\mathcal{F}_{1} \simeq_{p} \mathcal{F}_{2}$.

Step 3. $\mathbb{D}_{1}^{e}=\mathbb{D}_{2}^{e}$.

By contradiction. Let $\eta$ be a node such that $\eta \in \mathbb{D}_{1}^{e}$ and $\eta \notin \mathbb{D}_{2}^{e}$. Let $j_{0} \in \mathcal{J}_{1}(\eta)$, there exists $\xi \in \eta^{+}$such that $V_{\xi}^{1, j_{0}}(p) \neq 0$ because there are no trivial assets in our financial structures and all the assets of $\mathcal{F}_{1}$ are short-term. Since $\eta \notin \mathbb{D}_{2}^{e}$ and all the assets of $\mathcal{F}_{2}$ are short-term, we have for each $j \in \mathcal{J}_{2}, V_{\xi^{\prime}}^{2, j}(p)=0$ for all $\xi^{\prime} \in \eta^{+}$. Let $\lambda=\left(\lambda_{\xi}\right)_{\xi \in \mathbb{D}} \in \mathbb{R}_{++}^{\mathbb{D}}$ be a state price and let $\left(q^{1}, q^{2}\right) \in \mathbb{R}^{\mathcal{J}_{1}} \times \mathbb{R}^{\mathcal{J}_{2}}$ be the couple of arbitrage free prices such that $W^{1}\left(p, q^{1}\right) \lambda=0$ and $W^{2}\left(p, q^{2}\right) \lambda=0$. Since $W^{1 j_{0}}\left(p, q^{1}\right) \in \operatorname{im} W^{1}\left(p, q^{1}\right)=\operatorname{im} W^{2}\left(p, q^{2}\right)$, there exists $\zeta_{2}=\left(\zeta_{2}^{j}\right)_{j \in \mathcal{J}_{2}} \in \mathbb{R}^{\mathcal{J}_{2}}$ such that $W^{1 j_{0}}\left(p, q^{1}\right)=\sum_{j \in \mathcal{J}_{2}} W^{2}\left(p, q^{2}\right) \zeta_{2}^{j}$. So for all $\beta \in \mathbb{D} W_{\beta}^{1 j_{0}}\left(p, q^{1}\right)=$ $\sum_{j \in \mathcal{J}_{2}} W_{\beta}^{2}\left(p, q^{2}\right) \zeta_{2}^{j}$. So,

$$
\sum_{\xi^{\prime} \in \xi^{+} \cup\{\xi\}}\left[\lambda_{\xi^{\prime}} W_{\xi^{\prime}}^{1, j_{0}}\left(p, q_{1}\right)\right]=\sum_{\xi^{\prime} \in \xi^{+} \cup\{\xi\}}\left[\sum_{j \in \mathcal{J}_{2}} \lambda_{\xi^{\prime}} W_{\xi^{\prime}}^{2, j}\left(p, q^{2}\right) \zeta_{2}^{j}\right]
$$

Since $\xi^{-}=\eta=\xi\left(j_{0}\right)$ and all the assets are short-term, $W_{\xi^{\prime}}^{1, j_{0}}\left(p, q_{1}\right)=0$ for all $\xi^{\prime} \in \xi^{+}$. Since $\xi^{-}=\eta \notin \mathbb{D}_{2}^{e}$ and all the assets are short-term, $W_{\xi^{\prime}}^{2, j}=0$ for all $j \notin \mathcal{J}_{2}(\xi)$ and for all $\xi^{\prime} \in \xi^{+}$. Hence, one gets:

$$
0 \neq \lambda_{\xi} V_{\xi}^{1, j_{0}}(p)=\sum_{\xi^{\prime} \in \xi^{+} \cup\{\xi\}}\left[\sum_{j \in \mathcal{J}_{2}(\xi)} \lambda_{\xi^{\prime}} W_{\xi^{\prime}}^{2, j}\left(p, q^{2}\right) \zeta_{2}^{j}\right] .
$$

If $\xi \in \mathbb{D}_{2}^{e}$, we have

$$
\begin{gathered}
\sum_{\xi^{\prime} \in \xi^{+} \cup\{\xi\}}\left[\sum_{j \in \mathcal{J}_{2}(\xi)} \lambda_{\xi^{\prime}} W_{\xi^{\prime}}^{2, j}\left(p, q^{2}\right) \zeta_{2}^{j}\right]= \\
-\sum_{j \in \mathcal{J}_{2}(\xi)} \lambda_{\xi} q_{j}^{2} \zeta_{2}^{j}+\sum_{\xi^{\prime} \in \xi^{+}}\left[\sum_{j \in \mathcal{J}_{2}(\xi)} \lambda_{\xi^{\prime}} V_{\xi^{\prime}}^{2, j}(p) \zeta_{2}^{j}\right]=
\end{gathered}
$$

$\sum_{j \in \mathcal{J}_{2}(\xi)}\left[-\lambda_{\xi} q_{2}^{j}+\sum_{\xi^{\prime} \in \xi^{+}} \lambda_{\xi^{\prime}} V_{\xi^{\prime}}^{2, j}(p)\right] \zeta_{2}^{j}=0$ thanks to the no-arbitrage condition on $q_{2}$.

If $\xi \notin \mathbb{D}_{2}^{e}$, this implies that $\mathcal{J}_{2}(\xi)=\emptyset$ so

$$
\sum_{j \in \mathcal{J}_{2}(\xi)} \lambda_{\xi^{\prime}} W_{\xi^{\prime}}^{2, j}\left(p, q^{2}\right) \zeta_{2}^{j}=0, \forall \xi^{\prime} \in \xi^{+} \cup\{\xi\}
$$
0 .

In both cases we have: $\sum_{\xi^{\prime} \in \xi^{+} \cup\{\xi\}}\left[\sum_{j \in \mathcal{J}_{2}(\xi)} \lambda_{\xi^{\prime}} W_{\xi^{\prime}}^{2, j}\left(p, q^{2}\right) \zeta_{2}^{j}\right]=0$, which is in contradiction with $V_{\xi}^{1, j_{0}}(p) \neq$ 
Step 4. $\operatorname{im} V^{1}(p)=\operatorname{im} V^{2}(p)$.

Let $y \in \operatorname{im} V^{1}(p)$. There exists $z_{1} \in \mathbb{R}^{\mathcal{J}_{1}}$ such that $y=V^{1}(p) z^{1}$. Since $\mathcal{F}_{1} \simeq_{p} \mathcal{F}_{2}$, im $W^{1}\left(p, q^{1}\right)=$ $\operatorname{im} W^{2}\left(p, q^{2}\right)$. Hence, there exists $z^{2} \in \mathbb{R}^{\mathcal{J}_{2}}$ such that $\hat{y}=W^{1}\left(p, q^{1}\right) z^{1}=W^{2}\left(p, q^{2}\right) z^{2}$. We end the proof by showing that $y=V^{1}(p) z^{1}=V^{2}(p) z^{2}$, which implies that $y \in \operatorname{im} V^{2}(p)$ and consequently $\operatorname{im} V^{1}(p) \subset \operatorname{im} V^{2}(p)$. By a symmetric argument, one proves that im $V^{2}(p) \subset \operatorname{im} V^{1}(p)$.

For $\xi \in \mathbb{D}_{T}, y_{\xi}=\hat{y}_{\xi}=V_{\xi}^{1}(p) z_{1}=V_{\xi}^{2}(p) z_{2}$ because $V_{\xi}^{s}(p)=W_{\xi}^{s}\left(p, q_{s}\right)$ for all $\xi \in \mathbb{D}_{T}$ (with $\left.s \in\{1,2\}\right)$.

Let $\xi \in \mathbb{D}_{T-1}$. If $\xi \notin \mathbb{D}^{e}$ we still have: $y_{\xi}=\hat{y}_{\xi}=V_{\xi}^{1}(p) z^{1}=V_{\xi}^{2}(p) z^{2}$. If $\xi \in \mathbb{D}_{T-1} \cap \mathbb{D}^{e}, \hat{y}_{\xi}=W_{\xi}^{1}\left(p, q^{1}\right) z_{1}=$ $V_{\xi}^{1}(p) z_{1}-\sum_{j \in \mathcal{J}_{1}(\xi)} q_{j}^{1} z_{1}^{j}$ and $\hat{y}_{\xi}=W_{\xi}^{2}\left(p, q^{2}\right) z_{2}=V_{\xi}^{2}(p) z_{2}-\sum_{j \in \mathcal{J}_{2}(\xi)} q_{j}^{2} z_{2}^{j}$.

So, since $q^{1}$ and $q^{2}$ are arbitrage free,

$$
\begin{aligned}
\sum_{j \in \mathcal{J}_{1}(\xi)} q_{j}^{1} z_{1}^{j} & =\frac{1}{\lambda_{\xi}} \sum_{j \in \mathcal{J}_{1}(\xi)}\left[\left[\sum_{\xi^{\prime} \in \xi^{+}} \lambda_{\xi^{\prime}} V_{\xi}^{1, j}(p)\right] z_{1}^{j}\right] \\
& =\frac{1}{\lambda_{\xi}} \sum_{\xi^{\prime} \in \xi^{+}} \lambda_{\xi^{\prime}}\left[\sum_{j \in \mathcal{J}_{1}(\xi)} V_{\xi}^{1, j}(p) z_{1}^{j}\right] \\
& =\frac{1}{\lambda_{\xi}} \sum_{\xi^{\prime} \in \xi^{+}} \lambda_{\xi^{\prime}}\left[\sum_{j \in \mathcal{J}_{2}(\xi)} V_{\xi}^{2, j}(p) z_{2}^{j}\right]
\end{aligned}
$$

The last equality comes from the fact that for all $\xi^{\prime} \in \xi^{+}, \xi^{\prime} \in \mathbb{D}_{T}$ and then $V_{\xi}^{1}(p) z_{1}=V_{\xi}^{2}(p) z_{2}$ from the previous step. Hence,

$$
\sum_{j \in \mathcal{J}_{1}(\xi)} q_{j}^{1} z_{1}^{j}=\sum_{j \in \mathcal{J}_{2}(\xi)} q_{j}^{2} z_{2}^{j}
$$

which implies that $V_{\xi}^{1}(p) z^{1}=V_{\xi}^{2}(p) z^{2}$ and consequently for all $\xi \in \mathbb{D}_{T-1}, V_{\xi}^{1}(p) z^{1}=V_{\xi}^{2}(p) z^{2}$. A continuation of the same reasoning by backward induction leads to $V_{\xi}^{1}(p) z^{1}=V_{\xi}^{2}(p) z^{2}$ for all $\xi \in \mathbb{D}$, that is, $y=V^{1}(p) z^{1}=$ $V^{2}(p) z^{2}$.

\section{References}

[1] L. Angeloni and B. Cornet. Existence of financial equilibria in a multi-period stochastic economy., 8:1-31, 2006. Mathematical Economics, 8 (2006) 1-31.

[2] Z. Aouani and B. Cornet. Existence of financial equilibria with restricted participations. Journal of Mathematical Economics, 45: 772-786, 2009.

[3] J.-M. Bonnisseau and A. Chéry. On the equivalence of financial structures with long-term assets. Working Paper, Université Paris 1, 2013.

[4] B. Cornet and A. Ranjan. A remark on arbitrage free prices in multi-period economy. Working Paper, Université Paris 1, 2013.

[5] M. Magill and M. Quinzii. Theory of Incomplete Markets. Cambridge, 1996. Cambridge, (1996). 